\title{
Monitoring of Early and Late Age Hydration Products of Volcanic Ash Blended Cement Paste ${ }^{+}$
}

\author{
Antony Joseph *, Suad Al-Bahar, Jayasree Chakkamalayath, Amer Al-Arbeed and \\ Zakiya A. Rasheed
}

Construction and Building Materials Program, Energy and Building Research Centre, Kuwait Institute for Scientific Research, P.O. Box 24885, Safat, Kuwait; sbahar@kisr.edu.kw (S.A.-B.); jchakkamalayath@kisr.edu.kw (J.K.); aabeed@kisr.edu.kw (A.A.-A.); zrashid@kisr.edu.kw (Z.A.R.)

* Correspondence: ajoseph@kisr.edu.kw

+ Presented at the 1st International Conference on Smart Materials for Sustainable Construction-SMASCO 2019, Luleå, Sweden, 10-12 December 2019.

Published: 18 November 2019

\begin{abstract}
One of the major concerns of concrete industries is to develop materials that consume less natural virgin resources and energy to make sustainable construction practices. Efforts have been made and even implemented to use the waste/by product materials such as fly ash, slag, silica fume, and natural pozzolana as a partial or complete replacement for Portland cement in concrete mixtures. The deterioration of concrete structures in the existing hot and cold climates of Gulf Cooperation Council countries, along with chloride and sulphate attack, demands the use of pozzolanic materials for concrete construction. Volcanic ash incorporated cement based concretes are known for its better performance in terms of strength and durability in harsh marine environments. Understanding the cement hydration process and characterizing the hydration products in microstructural level is a complex and interdependent process that allows one to design complex mix proportions to produce sustainable concrete materials. In this paper, the early and late age hydration behavior along with micro- and pore structure of cement paste samples prepared with locally available ordinary Portland cement (OPC) and volcanic ash (VA) obtained from Saudi Arabia was monitored using X-ray diffraction (XRD), scanning electron microscopy (SEM), thermogravimetric (TGA) and N2-Adsorption analysis. The hydration progress of cement paste samples with different combinations of OPC and VA $(0 \%, 15 \%, 25 \%$, and $35 \%)$ at a w/c ratio of 0.45 after 14, 28, and 90 days were discussed. The qualitative XRD and SEM of cement paste samples showed no new phases were formed during the course of hydration. The disappearance of portlandite with increase in VA content was due to both pozzolanic effect and dilution effect. This was further confirmed quantitatively by the TGA observations that the samples with VA contain less $\mathrm{Ca}(\mathrm{OH})_{2}$ compared to the control specimens. $\mathrm{N}_{2}$ adsorption experiments after 90 days of curing showed larger hysteresis as the VA content increases. The studies show that the incorporation of volcanic ash certainly contributes to the generation of C-S-H and hence the cement hydration progress, especially in the later ages through pozzolanic reactions. A 15-25\% volcanic ash blended cement paste samples showed compact and denser morphological features, which will be highly detrimental for the durability performances.
\end{abstract}

Keywords: cement hydration; microstructure; pozzolanic reaction; volcanic ash 